\title{
LONG-TERM FORECASTING OF DEATH IN THE NEONATAL ICU USING HEART RATE CHARACTERISTICS MONITORING AND CLINICAL DATA
}

J.R. Moorman ${ }^{1}$, W.A. Carlo ${ }^{2}$, J. Kattwinkel ${ }^{1}$, R.L. Schelonka ${ }^{2}$, P.J. Porcelli ${ }^{3}$, C.T. Navarrete ${ }^{4}$, E. Bancalari ${ }^{4}$, J.L. Aschner ${ }^{5}$, M.W. Walker ${ }^{6}$, J.A. Perez ${ }^{7}$, C. Palmer ${ }^{8}$, D.P. Wagner ${ }^{1}$, G.J. Stukenborg ${ }^{1}$, D.E. Lake ${ }^{1}$, T.M. $\mathrm{O}^{\prime} \mathrm{Shea}^{3}$

${ }^{1}$ University of Virginia, Charlottesville, VA, ${ }^{2}$ University of Alabama at Birmingham, Birmingham, $A L,{ }^{3}$ Wake Forest University, Winston-Salem, NC, ${ }^{4}$ University of Miami, Miami, FL, ${ }^{5}$ Vanderbilt University, Nashville, TN, ${ }^{6}$ Greenville Hospital System, Greenville, SC, ${ }^{7}$ Winnie Palmer Children's Hospital, Orlando, FL, ${ }^{8}$ Pennsylvania State University, Hershey, PA, USA

Background: Early and on-going prediction of in-NICU mortality can aid clinicians and parents who are caring for high-risk preterm infants.

We developed heart rate characteristics (HRC) monitoring based on findings of reduced variability and transient decelerations prior to signs of sepsis. The HRC index, or HeRO score, is the fold-increase in risk of sepsis in the next 24 hours. Rising HeRO scores prompt clinicians to evaluate infants not otherwise suspected of illness. In the HeRO trial, we randomized 3000 VLBW infants in 9 US NICUs either to display of HeRO scores or to conventional monitoring. Those whose HeRO score results were displayed had statistically significant mortality reduction in excess of $20 \%$.

Aims: In this work, our aim was to test the hypothesis that average HeRO scores and knowledge of sepsis and ventilation can accurately predict NICU mortality from early in the stay.

Methods: We modeled 152 deaths in 1489 VLBW infants who received only conventional monitoring in the HeRO trial using multivariable logistic regression.

Results: In order of significance, the average HeRO score, presence of ventilator, birth weight, Apgar score at 5 (but not 1) minutes, and sepsis within the past 10 days predicted mortality ( $p<0.005$ for each). The model had good discrimination, with ROC area near 0.85 starting at 3 hours of monitoring.

Conclusions: Heart rate characteristics monitoring and clinical data about ventilation and sepsis predict NICU death beginning at 3 hours. These findings may add to the mortality benefit of HeRO monitoring. 